\title{
Grupo InterHAD: Interação Humano Artefato Digital
}

\author{
José Valderlei da Silva \\ Institute of Computing \\ University of Campinas \\ São Paulo, Brazil \\ vander.silva@ic.unicamp.br
}

\author{
Marleny Luque Carbajal \\ Institute of Computing \\ University of Campinas \\ São Paulo, Brazil \\ marleny.carbajal@students.ic.unicamp.br
}

\author{
M. Cecília C. Baranauskas \\ Institute of Computing \\ University of Campinas \\ São Paulo, Brazil \\ cecilia@ic.unicamp.br
}

\section{RESUMO}

O grupo de pesquisa InterHAD está vinculado ao Instituto de Computação da Universidade Estadual de Campinas (UNICAMP), e sua origem se deu nos anos 90 . O grupo adota uma postura subjetivista e reconhece a natureza situacional do design. Tem contribuído na formação de pesquisadores e na investigação científica de ponta, de maneira a promover o design socialmente consciente e o codesign. A produção do grupo inclui publicações e organização de eventos científicos, artefatos tecnológicos, registros de patentes, e tem sido agraciado com diversos prêmios.

\section{PALAVRAS-CHAVE}

Design Socialmente Consciente, Semiotica Organizacional, Desig Participativo

\section{CONHECENDO O GRUPO INTERHAD}

O grupo de pesquisa InterHAD (Interação Humano Artefato Digital) foi criado pelos alunos orientados pela Profa. Dra. M. Cecília C. Baranauskas, do Instituto de Computação (IC) da UNICAMP. Atualmente o grupo inclui também alunos do Prof. Dr. Rodrigo Bonacin e Prof. Dr. Julio Cesar dos Reis (ambos foram orientados pela Profa. Cecília). Além disso, diversos outros professores e pesquisadores compõem o InterHAD.

No InterHAD, entendemos o design como um processo social que não se restringe à tecnologia digital, mas que engloba também toda a sociedade na qual a demanda pelas tecnologias se inicia e se transforma continuamente. Em vez de considerar uma realidade objetiva e bem definida, buscamos explorar o contraste de diferentes pontos de vista para caracterizar a situação de design e levantar propostas de soluções para uma sociedade rica em diversidade cultural. A sociedade

Permission to reproduce or distribute, in whole or in part, material extracted from this work, verbatim, adapted or remixed, as well as the creation or production from the content of such work, is granted without fee for noncommercial use, provided that the original work is properly credited.

IHC 2019 - Trilha Fórum dos Grupos De Pesquisa, Outubro 21-25, 2019, Vitória, Brasil. In Anais Estendidos do XVIII Simpósio Brasileiro sobre Fatores Humanos em Sistemas Computacionais. Porto Alegre: SBC.

(c) 2019 by the author(s), in accordance with the terms of the Creative Commons Attribution-NonCommercial 4.0 International Public License (CC BY-NC 4.0). é composta por pessoas com habilidades e necessidades diversas, e o design, como o InterHAD entende, deve considerar as diferenças, sem segregação. Adotando uma postura subjetivista e reconhecendo a natureza situacional do design, o grupo reconhece e valoriza aspectos interpretativos, sociais e comunicativos no design de sistemas interativos [3]. Design, para nós, envolve a produção e interpretação de significado por todas as partes interessadas, que ocorre por meio de um processo iterativo e interativo de criação e interpretação de signos. Esse processo está aninhado em uma estrutura na qual aspectos informais, formais e técnicos coexistem em um ciclo de design socialmente consciente [1] inspirado em conceitos e artefatos da Semiótica Organizacional [4-6].

\section{Integrantes e Parceiros}

Além dos professores já mencionados, fazem parte do InterHAD: aluno de graduação (Rafael Eiki Matheus Imamura); alunas de mestrado (Andressa Cristina dos Santos, Geovana Evelyn Espinoza Taype, Yusseli Lizeth Méndez Mendoza); alunos de doutorado (Eliana Alves Moreira, Eliane Zambon Victorelli, Emanuel Felipe Duarte, Fabrício Matheus Gonçalves, Felipe Rodrigues Jensen, José Valderlei da Silva, Luã Marcelo Muriana, Marleny Luque Carbajal) e pesquisadores doutores (Vanessa Regina Margareth Lima Maike).

Pesquisadores que trabalham em parceria com o InterHAD, nucleando seus próprios grupos de pesquisa: Dra. Lara Piccolo (Open University, Reino Unido), Prof. Dr. Roberto Pereira (UFPR), Prof. Dr. Leonardo Cunha de Miranda (UFRN), Prof. Dr. Ig Ibert Bittencourt (UFAL), Prof. Dr. João Vilhete Viegas D'Abreu (NIED-UNICAMP), Prof. Dr. José Armando Valente (IA-UNICAMP), Dr. Ricardo Caceffo (IC-UNICAMP), Profa. Dra. Maria Teresa Mantoan (FE-UNICAMP), Dra. Tânia Cristina Lima e Prof. Dr. Rodrigo Bonacin (CTI Renato Archer), e Prof. Dr. Julián Esteban Gutiérrez Posada (Uniquindío, Colômbia), entre outros.

\section{Prêmios}

O trabalho desenvolvido por membros do InterHAD foi reconhecido em diversos prêmios relevantes nacionais e internacionais. Dentre os principais, a Profa. M. Cecília C. Baranauskas recebeu em 2010 o ACM Rigo Award por suas contribuições ao design da comunicação em IHC. Em 2015, ela foi premiada pela Comissão Brasileira de IHC por suas 
contribuições científicas na comunidade. Outros prêmios são listados em nosso portal ${ }^{1}$.

A seguir, descrevemos três pilares: formação de pessoas, pesquisa e contribuições científicas com impacto social.

\section{FORMAÇÃO DE PESSOAS}

No InterHAD valorizamos não apenas a obtenção do título de graduado ou pós-graduado, mas a formação de pessoas com pensamento crítico. Pautados pelos valores do InterHAD, muitos ex-alunos agora estão nucleando seus próprios grupos de pesquisa em várias partes do Brasil (UFPR, UTFPR, Unipampa, UFRN, CTI Renato Archer, IBM Research, UFAL, UFSCar, FACCAMP, IFSP, UNICAMP-FT, Uninove) e do mundo (Uniquindío e Universidade de Manizales na Colômbia, e Open University no Reino Unido).

\section{PESQUISA}

O grupo tem desenvolvido trabalhos de investigação no contexto de diversos projetos de pesquisa financiados por agências de fomento nacional.

\section{Projetos Atuais}

Sistemas Socioenativos: Investigando Novas Dimensões no Design da Interação Mediada por Tecnologias de Informação e Comunicação. Projeto temático FAPESP \#2015/16528-0 (em andamento). O projeto visa criar frameworks conceituais que articulem o uso de tecnologias de interfaces vestíveis, tangíveis e naturais com o conceito de enação para o design socialmente consciente em diferentes contextos. Espera-se que o projeto possa informar o design de sistemas que estamos chamando de socioenativos, nos quais novas formas de interação e novos fatores (emocionais, físicos e culturais) se somam à ubiquidade e às redes sociais, criando novos desafios. Internet das Coisas (Humanas), instalações artísticas acessíveis, interações naturais, pensamento computacional transparente nas escolas, são alguns dos temas explorados e em breve estarão nos anais das principais conferências e revistas da área.

OpenDesign: Técnicas e Artefatos para o Design Socialmente Consciente de Sistemas Computacionais. Projeto FAPESP \# 2015/24300-9 (em andamento). Este projeto objetiva caracterizar o conceito de OpenDesign e formalizar um modelo de design socialmente consciente, orientado em torno dos princípios de colaboração aberta, do igualitarismo, da meritocracia, e da auto organização, no processo de design de sistemas.

\section{Projetos de pesquisa desenvolvidos}

Nos últimos anos, diversos projetos de pesquisa foram conduzidos pelos membros do InterHAD. O grupo conta com a

\footnotetext{
${ }^{1}$ https://interhad.nied.unicamp.br/
}

infraestrutura do IC-UNICAMP e também do NIED, o Núcleo de Informática aplicada à Educação, da UNICAMP. O grupo trabalha de forma colaborativa para a construção de resultados significativos e de impacto científico e social. Dentre os projetos concluídos mais relevantes, podemos destacar:

AbINV (2011 - 2014). Aprendizagem Baseada na Investigação (AbINV) é uma Instância do projeto UCA-UNICAMP-CNPq: "O Laptop Educacional e a Educação Baseada na Investigação: do estudo de fatos científicos para o fazer científico"(\#550382 /2011-9), coordenado pelo Prof. Dr. José Armando Valente. O objetivo foi estudar a implantação de uma abordagem pedagógica baseada na investigação, onde professores e alunos pudessem estar engajados em "fazer ciência"usando características próprias do laptop educacional.

EcoWeb (2010 - 2013). O projeto EcoWeb - Design de Interação na Web Social Inclusiva foi financiado pelo CNPq (processo \#560044/2010) para pesquisar e promover a construção de sistemas de inclusão social. O projeto situava-se no contexto do Desafio \#4 da Sociedade Brasileira de Computação: "Acesso Participativo e Universal do Cidadão Brasileiro ao Conhecimento". Como resultado, diversos artefatos como frameworks conceituais e instrumentos de design foram publicados e eles vêm até hoje auxiliando outros pesquisadores no processo de design consciente e orientado a valores.

XO na Escola e Fora Dela (2010 - 2013). Projeto financiado pelo CNPq \#475105/2010-9. O objetivo deste projeto foi desenvolver um modelo de implantação de laptops educacionais em escolas públicas. $O$ projeto se beneficiou de uma doação de mais de 500 laptops XO, que foram, por sua vez, doados para uma escola de ensino fundamental localizada na periferia de Campinas. O modelo foi construído juntamente com as partes interessadas (alunos, profissionais da educação e comunidade escolar). O livro "XO na escola" [2] narra todo o processo e lições aprendidas com o projeto.

Todos Nós em Rede (2009 - 2011). Redes Sociais e Autonomia Profissional - Novos Rumos para Formação Continuada à Distância de Professores de AEE (Atendimento Educacional Especializado). Esse é o projeto financiado pelo Proesp/CAPES 2009 (\# 23038.01457/2009-11), que objetiva a formação continuada a distância de professores de AEE dos sistemas de ensino público brasileiro, por meio da constituição de redes sociais inclusivas desses profissionais. A rede social visa: a) buscar expertise para a solução de problemas que encontram no cotidiano em salas de recursos multifuncionais; b) compartilhar ideias de casos bem sucedidos e casos ainda não resolvidos; c) estabelecer uma dinâmica continuada de estudos e respostas mediadas pela tecnologia. O sistema Todos Nós em Rede vem sendo construído e utilizado por professores de AEE e demais participantes do projeto, contribuindo para o aprimoramento e melhoria da qualidade de ensino. 
e-Cidadania (2007 - 2010). Financiado pelo Virtual Research Institute FAPESP - Microsoft Research, o "e-Cidadania: Sistemas e Métodos na Constituição de uma Cultura mediada por Tecnologias de Informação e Comunicação" teve por objetivo estudar e propor soluções aos desafios do design da interação e interface de usuário em sistemas relacionados ao contexto do exercício de cidadania, a partir do desenvolvimento de ações conjuntas com a comunidade-alvo. Além dos benefícios materiais e imateriais recebidos pela comunidade parceira de Campinas, o resultado prático da pesquisa foi a construção de uma rede social inclusiva, o VilaNaRede, e a publicação do livro "Codesign de Redes Digitais"[3] .

\section{CONTRIBUIÇÕES}

\section{Livros em Destaque}

- 2016: Socially Aware Organisations and Technologies: Impact and challenges [5]

- 2013: Codesign de Redes Digitais: tecnologia e educação a serviço da inclusão social [3]; XO na escola: construção compartilhada de conhecimento - lições aprendidas [2]

- 2013: XO na escola: construção compartilhada de conhecimento - lições aprendidas [2]

- 2000, 2003: Design e Avaliação de Interfaces HumanoComputador (primeiro livro de IHC em língua portuguesa lançado no Brasil) [7] .

\section{Organização de conferências}

- 17th International Conference on Informatics and Semiotics in Organisations - ICISO 2016: "Socially Aware Organisations and technologies: impact and challenges".

- II Congresso Brasileiro de Informática na Educação - CBIE 2013: "Informática na Educação: da pesquisa à ação".

- 9th International Conference on Informatics and Semiotics in Organisations - ICISO 2006: "Interfacing Society, Technology and Organisations".

- II Workshop sobre Fatores Humanos em Sistemas Computacionais - IHC'99: "Rompendo barreiras entre pessoas e computadores".

\section{Produção Científica e Tecnológica}

Publicações. Apartir do ano 2000, foram: 9 livros, 70 capítulos de livros, mais de 260 artigos em conferências, 54 artigos em revistas, 25 artigos curtos e mais de 45 relatórios técnicos.

\section{Artefatos Tecnológicos.}

- SobraPet: ambientes IoT para crianças no contexto hospitalar

- TangiTime: artefato interativo que permite explorar a passagem do tempo no mundo de forma tangível

- Tan2Talk: ambiente tangível e lúdico para comunicação

- TaPrEC: ambiente tangível de baixo custo para ensinar conceitos de programação para crianças
- DSC: sistema web colaborativo para clarificação de problemas baseado em Design Socialmente Consciente

- Quid: suporte a revisões bibliográficas sistemáticas

- CPES: Collaborative Programmable Environment for Storytelling

- AIR - Adjustable Interactive Rings for iDTV

- GWIDO: Game With an Interaction Design Purpose

- Todos Nós em Rede: rede social para professores de AEE

Artefatos de Design e Frameworks.

- Heurísticas para Natural User Interfaces

- VCIA: Abordagem Orientada a Valores e Informada Culturalmente

- BME: autoria de jogos educacionais estilo RPG

- Design para Afetibilidade

- Socially-informed Energy Eco-feedback Technology

\section{INFRAESTRUTURA DISPONÍVEL PARA A EXECUÇÃO DAS PESQUISAS}

O grupo é vinculado ao IC-UNICAMP, contando com apoio de uma das melhores universidades do país e de toda equipe administrativa e técnica do Instituto. Em 2019 fomos contemplados com um laboratório que está sendo estruturado com ferramentas que darão suporte às pesquisas desenvolvidas pelos membros do InterHAD. O grupo mantém parceria com o NIED-UNICAMP em propostas e execução de pesquisas, assim contamos com servidores, salas e suporte do Núcleo.

\section{INTERESSES EM PARCERIAS}

O InterHAD está de portas abertas para colaborar com outros grupos de pesquisa e pessoas que tenham audácia e coragem para enfrentar os desafios das transformações em IHC; em particular, nosso interesse atual envolve o OpenDesign e a interação em sistemas socioenativos.

\section{REFERÊNCIAS}

[1] Maria Cecília Calani Baranauskas et al. 2014. Social awareness in HCI. Interactions 21, 4 (2014), 66-69.

[2] Maria Cecília Calani Baranauskas, Maria Cecília Martins, and R. de Assis. 2012. XO na escola e fora dela: uma proposta semio-participativa para tecnologia, educação e sociedade. (2012).

[3] Maria Cecília Calani Baranauskas, Maria Cecília Martins, and José Armando Valente. 2013. Codesign de Redes Digitais: tecnologia e educação a serviço da inclusão social. Penso Editora.

[4] Kecheng Liu. 2000. Semiotics in information systems engineering. Cambridge University Press.

[5] Kecheng Liu and Weizi Li. 2014. Organisational semiotics for business informatics. Routledge.

[6] Ronald K. Stamper, R. A. Meersman, and R. P. Van de Riet. 1992. Language and computing in organised behaviour. Linguistic instruments in knowledge engineering (1992), 143-163.

[7] Heloisa Vieira and Maria Cecília Calani Baranauskas. 2003. Design e avaliação de interfaces humano-computador. Creative Commons, Brasil. 